VOL. $26(1982), 321-330$.

\title{
AN AVERAGE DISTANCE RESULT IN EUCLIDEAN $n$-SPACE
}

\author{
John StRantzen
}

The following result has been established by Stadje and Gross:

THEOREM. If $\langle X, d\rangle$ is a compact connected metric space, then there is a unique positive reai number $a(\langle X, d\rangle)$ with the property that for each natural number. $n$, and for all $\left\{x_{1}, x_{2}, \ldots, x_{n}\right\} \subset X$, there exists $y \in X$ for which $\frac{1}{n} \sum_{i=1}^{n} d\left(x_{i}, y\right)=a(\langle X, d\rangle)$.

Stadje also proved that if $d$ is the Euclidean metric on $\mathbb{R}^{2}$, and $X$ is a compact convex subset of $\mathbf{R}^{2}$ then $a((X, d)) \leq(D / 2) \sqrt{5-2 \sqrt{3}}$, where $D$ is the diameter of $X$. Stadje further claimed that his proof of this result generalised to $\mathbb{R}^{n}$ giving the same bound, and conjectured that in $\mathbf{R}^{2}$, $D / \sqrt{3}$ is an upper bound for $a(\langle X, d\rangle)$.

In this paper we show that if $d$ is the Euclidean metric on $\mathbf{R}^{n}$, and $X$ is a compact convex subset of $\mathbf{R}^{n}$, then $a((x, d)) \leq D \sqrt{n /(2 n+2)}$ where $D$ is the diameter of $X$. We also show that if $X$ is the $k$ skeleton of a regular $n$ simplex $J$ (that is, $X$ is the union of all $k$ simplices whose vertices are vertices of $J$ ), and $D$ is the diameter of $X$,

Received 28 April 1982. The author wishes to thank Sid Morris for the interest he has generated among the La Trobe University Pure Mathematics staff on Stadje numbers. He also wishes to thank David Yost for references [1] and [3]. The author was unaware that a proof of Theorem 1 was in the literature, and, in fact produced an elementary but lengthy proof of Theorem 1 in attempting to improve on Stadje's erroneous estimate in [5]. 
then

$$
a(\langle x, d\rangle)=\frac{D}{(n+1) \sqrt{2 k+2}}((k+1) \sqrt{k}+(n-k) \sqrt{k+2}) .
$$

By putting $k=n$ this shows the bound $D \sqrt{n /(2 n+2)}$ is optimal. Further, since for $n \geq 4$ it follows that $\sqrt{n /(2 n+2)} \geq \frac{1}{2} \sqrt{5-2 \sqrt{3}}$, Stadje's claim that $(D / 2) \sqrt{5-2 \sqrt{3}}$ is an upper bound for $a(\langle X, d\rangle)$ in $\mathbf{R}^{n}$ is false for $n \geq 4$.

\section{A classical result}

THEOREM 0 . If $\langle X, d\rangle$ is a compact connected metric space then there is a unique positive real number $a(\langle X, d\rangle)$ with the property that for each natural number $n$, and for all $\left\{x_{1}, x_{2}, \ldots, x_{n}\right\} \subset X$, there exists $y \in X$ for which $(1 / n) \sum_{i=1}^{n} d\left(x_{i}, y\right)=a(\langle X, d\rangle)$.

For a proof of Theorem 0 see [2] or [5] (where a more general result is proved with $X$ being a compact connected Hausdorff space and $d: X \times X \rightarrow \mathbb{R}$ being a continuous and symetric function).

DEFINITION 1. Let $\langle X, d\rangle$ be a compact connected metric space. The number $a(\langle X, d\rangle)$ defined in Theorem 0 shall be called the Stadje number of $\langle X, d\rangle$. If $D$ is the diameter of $X$ the number $a(\langle x, d\rangle) / D$ is called the dispersion constant of $\langle X, d\rangle$ and is denoted $m(X, d)$.

We now quote a classical result first published in 1901 (see [3]) which leads quickly to an upper bound for stadje numbers for bounded closed convex subsets of $\mathbb{R}^{n}$ (with Euclidean metric) which is the central purpose of this paper.

THEOREM 1. Let $\mathbf{R}^{n}$ be real Euclidean n-space, and $X$ a bounded subset of $\mathbf{R}^{n}$ containing at least two points. There exists a unique closed ball $B$ of smallest radius $r$ such that $X \subseteq B$, and if $D$ is the diameter of $X$ then $r \leq D \sqrt{n /(2 n+2)}$.

A simple proof of Theorem 1 appears in [1]. 
LEMMA 1. Let $X$ be a bounded closed convex subset of $\mathbb{R}^{n}$ containing at least two points, and let $B$ be the closed ball of smallest radius such that $X \subseteq B$. If $b$ is the centre of $B$ then $b \in X$.

Proof. This can be deduced from the results in [1], specifically Lemma 1, p. 772 and Property 1, p. 773. Alternatively a simple direct proof is as follows. Let $A$ be a closed ball in $\mathrm{R}^{n}$ of centre $a$ and radius $s$ such that $X \subseteq A$. We will show that if $a \notin X$ and $\delta>0$ is the distance of $a$ from $X$ then there is a closed ball $C$ of radius $\sqrt{s^{2}-\delta^{2}}<s$ such that $X \subseteq C$. Hence if the centre of $a$ is not in $X$ then $A$ is not the unique ball of smallest radius containing $X$. Since $a \notin X$ and $X$ is compact there is a point $c \in X$ such that $d(a, c)=\delta$. Let $a c$ be the line in $\mathbb{R}^{n}$ containing $a$ and $c$ and let $x \in X$. By consideration of the plane containing $\alpha, c$ and $x$ and Euclidean plane geometry we see that if the angle $(a c x$ is less than $\pi / 2$, since $d(a, x) \geq \delta=d(a, c)$, the point $y$ which is the projection of $a$ on the line $c x$ belongs to the segment $c x$ and thus to the convex set $X$. But $y$ is the point of $c x$ of shortest distance from $a$, and $y \neq c$ since $(a c x \neq \pi / 2$. Therefore $d(a, y)<d(a, c)=\delta$ which contradicts the fact that $\delta$ is the distance of $a$ from $X$. Hence < acx is greater than $\pi / 2$ from which it follows, again by Euclidean plane geometry that $d(a, x) \geq \sqrt{d(c, d)^{2}+d(a, c)^{2}}=\sqrt{d(c, x)^{2}+\delta^{2}}$. Since $x \in X \subseteq A$, $d(a, x) \leq s$. Hence $s^{2} \geq d(c, x)^{2}+\delta^{2}$ or $d(c, x)^{2} \leq s^{2}-\delta^{2}$. Thus if we let $C$ be the closed ball of centre $c$ and radius $\sqrt{s^{2}-\delta^{2}}$ then $x \in C$ or $X \subseteq C$. It is interesting to note that the reduction of the problem to Euclidean plane geometry works in the more general case where instead of $\mathbf{R}^{n}$ we take any inner product space (thus any real inner product space), and we take $X$ to be a convex compact subset of the inner product space. //

LEMMA 2. Let $X$ be a bounded closed convex subset of $\mathbb{R}^{n}$ and $D$ be the diameter of $X$. Then $a(\langle x, d\rangle) \leq D \sqrt{n /(2 n+2)}$, and if $X$ contains more than a single point $m(X, d) \leq \sqrt{n /(2 n+2)}$.

Proof. If $X=\{x\}$ then trivially $a(\langle X, d))=0$ so the result follows. If $X$ contains more than a single point, apply Theorem 1 to find 
the ball $B$ of smallest radius $r$ containing $X$. Let $b$ be the centre of $B$. By Lemma $1, b \in X$. Apply Theorem 0 to the subset $\{b\}$ of $X$ to obtain the existence of a point $y \in X$ for which $d(b, y)=a(\langle X, d\rangle)$ But $y \in X \subseteq B$, so $d(b, y) \leq r \leq D \sqrt{n /(2 n+2)}$. Hence $a(X, d) \leq D \sqrt{n /(2 n+2)}$ and $m(X, d) \leq \sqrt{n /(2 n+2)}$, as required.

In the next section we demonstrate the upper bound for stadje numbers so obtained is optimal.

\section{Dispersion constants for skeletons of regular simplices}

DEFINITION 2. Let $\left\{x_{1}, x_{2}, \ldots, x_{m+1}\right\} \subseteq \mathbb{R}^{n}$, where $m \leq n$ is such that $d\left(x_{i}, x_{j}\right)=D>0,0 \leq i<j \leq m+1$.

The m-simplex with vertices $x_{1}, x_{2}, \ldots, x_{m+1}$ is the convex hull of the set $\left\{x_{1}, x_{2}, \ldots, x_{m+1}\right\}$, and is denoted $J\left(x_{1}, x_{2}, \ldots, x_{m+1}\right)$

DEFINITION 3. If $J\left(x_{1}, x_{2}, \ldots, x_{m+1}\right)$ is an $m$ simplex in $\mathbf{R}^{n}$ and $1 \leq k \leq m$ then the k-skeleton of $J\left(x_{1}, x_{2}, \ldots, x_{m+1}\right)$ is the union of all the $k$-simplices whose vertices are in the set ' $\left\{x_{1}, x_{2}, \ldots, x_{m+1}\right\}$. It is denoted $J^{k}\left(x_{1}, x_{2}, \ldots, x_{m+1}\right)$.

For example $J\left(x_{1}, x_{2}, x_{3}\right)$ is a closed triangular region with vertices $x_{1}, x_{2}$ and $x_{3}$ and $J^{l}\left(x_{1}, x_{2}, x_{3}\right)$ is the triangle which forms the boundary of $J\left(x_{1}, x_{2}, x_{3}\right)$.

DEFINITION 4. The centroid of $J\left(x_{1}, x_{2}, \ldots, x_{m+1}\right)$ is the point $(1 /(m+1))\left(x_{1}+x_{2}+\ldots+x_{m+1}\right)$ of $J\left(x_{1}, x_{2}, \ldots, x_{m+1}\right)$.

It is clear that isometric spaces have the same Stadje numbers, and that if $J\left(x_{1}, \ldots, x_{m+1}\right)$ is an $m$ simplex in $\mathbf{R}^{n}$ where $d\left(x_{1}, x_{2}\right)=D$ and $z_{1}, z_{2}, \ldots, \tau_{n+1}$ is an orthonormal basis for $\mathbb{R}^{n+1}$, then the mapping $\phi: J\left(x_{1}, \ldots, x_{m+1}\right) \rightarrow J\left((D / \sqrt{2}) z_{1}, \ldots,(D / \sqrt{2}) z_{m+1}\right)$ given by 
$\phi\left(\sum_{i=1}^{n} t_{i} x_{i}\right)=(D / \sqrt{2}) \sum_{i=1}^{n} t_{i} \tau_{i}$ is a centroid preserving isometry. Further by restricting $\phi$ to $J^{k}\left(x_{1}, \ldots, x_{m+1}\right)$ we see $J^{k}\left(x_{1}, \ldots, x_{m+1}\right)$ and $J^{k}\left((D / \sqrt{2}) z_{1}, \ldots,(D / \sqrt{2}) z_{m+1}\right)$ are isometríic. It is also evident by scaling that the stadje number of $J\left((D / \sqrt{2}) \tau_{1}, \ldots,(D / \sqrt{2}) \tau_{m+1}\right)$ is $(D / \sqrt{2})$ multiplied by the stadje number of $J^{k}\left(z_{1}, \ldots, z_{m+1}\right)$. Thus we restrict our attention to simplices $J\left(l_{1}, \ldots, l_{m+1}\right)$ and their $k$-skeletons.

LEMMA 3. Let $x \in J\left(z_{1}, \ldots, z_{m+1}\right)$ and let $f(x)=\sum_{i=1}^{m+1} d\left(x, z_{i}\right)$. Then $f(x)$ is a minimum if $x$ is the centroid of $J\left(l_{1}, \ldots, z_{m+1}\right)$. Proof. If $x \in J\left(z_{1}, \ldots, z_{m+1}\right)$ then $x=\sum_{i=1}^{m} t_{i} z_{i}$ where $t_{i} \geq 0$ and $\sum_{i=1}^{m+1} t_{i}=1$ :

$$
\begin{aligned}
f(x) & =\sum_{i=1}^{m+1} d\left(x, z_{i}\right) \\
& =\sum_{i=1}^{m+1} \sqrt{\left(x-l_{i}\right) \cdot\left(x-\tau_{i}\right]} \\
& =\sum_{i=1}^{m+1}\left[\left(\sum_{j=1}^{m+1} t_{j}^{2}\right)+1-2 t_{i}\right]^{\frac{1}{2}}
\end{aligned}
$$

Thus we wish to minimise $\sum_{i=1}^{m}\left[\left(\sum_{j=1}^{m+1} t_{j}^{2}\right)+1-2 t_{i}\right]^{\frac{1}{2}}$ subject to $t_{i} \geq 0$, $i=1,2, \ldots, m+1$ and $\sum_{i=1}^{m+1} t_{i}=1$. By standard techniques the minimum is achieved for $t_{1}=t_{2}=\ldots=t_{m+1}=1 /(m+1)$, that is when $x$ is the centroid of $J\left(z_{1}, \ldots, z_{m+1}\right)$. //

LEMMA 4. Let $x=t_{1} z_{1}+t_{2} z_{2}+\ldots+t_{k+1} z_{k+1}, \quad 0 \leq t_{j} \leq t_{1}$, 
326

John Strantzen

$j=2,3, \ldots, k+1$ and $t_{1}+t_{2}+\ldots+t_{k+1}=1$. That is, $x \in J\left(z_{1}, z_{2}, \ldots, z_{k+1}\right)$ and the closest vertex to $x$ is $l_{1}$. Let $z$ be the centroid of $J\left(z_{i_{1}}, z_{i_{2}}, \ldots, z_{i_{k+1}}\right)$ where $\left\{z_{i_{1}}, \ldots, z_{i_{k+1}}\right\}$ is a $k+1$ element subset of $\left\{z_{1}, \ldots, z_{n+1}\right\}$. Then $d\left(z_{1}, z\right) \geq d(x, z)$.

Proof.

$$
\begin{aligned}
d\left(z_{1}, z\right) & =\sqrt{\left(z-z_{1}\right) \cdot\left(z-z_{1}\right)} \\
& =\left[\left(\frac{1}{k+1} \sum_{j=1}^{k+1} z_{i_{j}}-z_{1}\right) \cdot\left(\frac{1}{k+1} \sum_{j=1}^{k+1} z_{i_{j}}-z_{1}\right)\right]^{\frac{1}{2}} \\
& = \begin{cases}\sqrt{k /(k+1)}, & 1 \in\left\{i_{1}, \ldots, i_{k+1}\right\}, \\
\sqrt{(k+2) /(k+1)}, & 1 \leqslant\left\{i_{1}, \ldots, i_{k+1}\right\} .\end{cases}
\end{aligned}
$$

Suppose $\left\{z_{1}, \ldots, z_{k+1}\right\} \cap\left\{z_{i_{1}}, \ldots, z_{i_{k+1}}\right\}=\left\{z_{i_{1}}, \ldots, z_{i_{l}}\right\}$. Then

$$
d(x, z)=\left[\sum_{i=1}^{k+1} t_{i}^{2}+\frac{1}{k+1}-\frac{2}{k+1}\left(t_{i_{1}}+\ldots+t_{i_{i}}\right)\right]^{\frac{1}{2}} .
$$

Case 1. $1 \in\left\{i_{1}, \ldots, i_{2}\right\}$.

Without loss of generality put $i_{1}=1$;

$$
\begin{aligned}
d(x, z)^{2} & =\sum_{i=2}^{k+1} t_{i}^{2}+\frac{1}{k+1}-\frac{2 t}{k+1}+t_{1}^{2}-\frac{2}{k+1}\left(t_{i_{2}}+\ldots+t_{i_{i}}\right) \\
& \leq\left(\sum_{i=2}^{k+1} t_{i}\right)^{2}+\left(t_{1}-\frac{1}{k+1}\right)^{2}+\frac{k}{(k+1)^{2}} \\
& =\left(1-t_{1}\right)^{2}+\left(t_{1}-\frac{1}{k+1}\right)^{2}+\frac{k}{(k+1)^{2}} \\
& =\left(1-\frac{1}{k+1}\right)^{2}-2\left(1-t_{1}\right)\left(t_{1}-\frac{1}{k+1}\right)+\frac{k}{(k+1)^{2}} \\
& \leq\left(1-\frac{1}{k+1}\right)^{2}+\frac{k}{k+1}\left(\text { since } \frac{1}{k+1} \leq t_{1} \leq 1\right) \\
& =\frac{k}{k+1}=d\left(l_{1}, z\right)^{2} .
\end{aligned}
$$

https://doi.org/10.1017/S0004972700005815 Published online by Cambridge University Press 
Case 2. $1 \notin\left\{i_{1}, \ldots, i_{2}\right\}$ whence $1 \notin\left\{i_{1}, \ldots, i_{k+1}\right\}$.

$$
\begin{aligned}
d(x, z)^{2} & =\sum_{i=1}^{k+1} t_{i}^{2}+\frac{1}{k+1}-\frac{2}{k+1}\left(t_{i_{1}}+\ldots+t_{i_{i}}\right) \\
& \leq\left(\sum_{i=1}^{k+1} t_{i}\right)^{2}+\frac{1}{k+1} \\
& =\frac{k+2}{k+1} \\
& =d\left(I_{1}, z\right)^{2} .
\end{aligned}
$$

LEMMA 5. $a\left(\left\{J^{k}\left(z_{1}, \ldots, z_{m+1}\right), d\right\rangle\right)=\frac{1}{(m+1) \sqrt{k+1}}[(k+1) \sqrt{k+(m-k) \sqrt{k+2}}]$.

Proof. Write $X$ for $d^{k}\left(z_{1}, \ldots, z_{m+1}\right)$.

By Theorem 0 there is a $y \in X$ for which

$$
\frac{1}{m+1} \sum_{i=1}^{m+1} d\left(z_{i}, y\right)=a((x, d)) \text {. }
$$

By symmetry we may suppose $y \in J\left(z_{1}, \ldots, z_{k+1}\right)$. Let $z$ be the centroid of $J\left(z_{1}, \ldots, z_{k+1}\right)$.

By Lemma 3,

$$
\sum_{i=1}^{k+1} d\left(z_{i}, y\right) \geq \sum_{i=1}^{k+1} d\left(z_{i}, z\right)
$$

If $k+1<i \leq m+1, J\left(z_{1}, \ldots, z_{k+1}, z_{i}\right)$ is a $(k+1)$-simplex and the line $z \tau_{i}$ is perpendicular to the space $S$ determined by the points $\left\{z_{1}, z_{2}, \ldots, z_{k+1}\right\} \quad$ (because

$$
\left(z_{i}-z\right) \cdot\left(z_{t}-z_{1}\right)=\left(z_{i}-\frac{1}{k+1} \sum_{j=1}^{k+1} z_{j}\right) \cdot\left(z_{t}-z_{1}\right)=0
$$

if $0 \leq t \leq k+1$ ).

Thus as $y$ is in the space $S$,

$$
d\left(z_{i}, z\right) \leq d\left(z_{i}, y\right) \text { for } k+1<i \leq n+1 \text {. }
$$


By (i) and (ii),

$$
\sum_{i=1}^{m+1} d\left(z_{i}, y\right) \geq \sum_{i=1}^{m+1} d\left(z_{i}, z\right) .
$$

Thus

$$
\begin{aligned}
a(\langle x, d\rangle) & \geq \frac{1}{m+1} \sum_{i=1}^{m+1} d\left(z_{i}, z\right) \\
& =\frac{1}{m+1}\left(\sum_{i=1}^{k+1} d\left(z_{i}, z\right)_{i}+\sum_{i=k+2}^{m+1} d\left(z_{i}, z\right)\right) .
\end{aligned}
$$

But, as has been seen in the proof of Lema 3 ,

$$
d\left(z_{i}, z\right)= \begin{cases}\sqrt{k /(k+1)}, \quad 1 \leq i \leq k+1, \\ \sqrt{(k+2) /(k+1)}, \quad k+1<i \leq m+1 .\end{cases}
$$

Thus

(iv)

$$
a(\langle x, d\rangle) \geq \frac{1}{(m+1) \sqrt{k+1}}[(k+1) \sqrt{k}+(m-k) \sqrt{k+2}] .
$$

Let $z_{1}, z_{2}, \ldots, z_{s}$ be those points of $X$ which are centroids of $k$-subsimplices of $X$. There are ${ }^{m+1} C_{k+1}$ such $k$-subsimplices, so $s={ }^{m+1} C_{k+1}$

By Theorem 0 , there is a $y \in X$ for which

$$
\frac{1}{s} \sum_{i=1}^{s} d\left(z_{i}, y\right)=a(\langle x, d\rangle) \text {. }
$$

By symmetry we may suppose $y \in J\left(z_{1}, \ldots, z_{k+1}\right)$, and that $y$ is closest to $z_{1}$ (that is, $y=t_{1} z_{1}+t_{2} z_{2}+\ldots+t_{k+1} z_{k+1}$, where $0 \leq t_{j} \leq t_{1}$ for $j=2,3, \ldots, k+1$ and $t_{1}+t_{2}+\ldots+t_{k+1}=1$ ).

Now if $z$ is the centroid of any $k$ subsimplex of $X$, by Lemma 4 , $d\left(z_{1}, z\right) \geq d(y, z)$.

Thus 


$$
\begin{aligned}
& \text { Average distance in Euclidean } n \text {-space } \\
& a(\langle x, d\rangle)=\frac{1}{s} \sum_{i=1}^{s} d\left(z_{i}, y\right) \leq \frac{1}{s} \sum_{i=1}^{s} d\left(z_{i}, z_{1}\right) .
\end{aligned}
$$

Again if $z_{i}$ is the centroid of a $k$-subsimplex with vertex $\tau_{1}$, $d\left(z_{i}, z_{1}\right)=\sqrt{k /(k+1)}$, and if $z_{i}$ is the centroid of a-k-subsimplex which does not have vertex $l_{1}, d\left(z_{i}, l_{1}\right)=\sqrt{(\underset{z}{k}+2) /(k+1)}$. Further, as there are ${ }^{m_{C_{k}}}{ }_{k}$-subsimplices with vertex $Z_{1}$, and ${ }^{m+1} C_{k+1}-{ }^{m} C_{k}$ other subsimplices, it follows that

$$
\begin{aligned}
a((x, d)) & \leq \frac{1}{{ }^{m+1} C_{k+1}}\left[{ }^{m} C_{k} \sqrt{k /(k+1)}+\left({ }^{m+1} C_{k+1}-{ }^{m} C_{k}\right) \sqrt{(k+2) /(k+1)}\right] \\
& =\frac{1}{(m+1) \sqrt{k+1}}[(k+1) \sqrt{k+(m-k) \sqrt{k+2}}] .
\end{aligned}
$$

The lemma is established by (iv) and ( $v$ ). //

This leads to the two main-results in this paper, namely:

THEOREM 2.

$$
a\left(\left\langle J^{k}\left(x_{1}, \ldots, x_{m+1}\right), d\right\rangle\right)=\frac{D}{(m+1) \sqrt{2 k+2}}[(k+1) \sqrt{k}+(m-k) \sqrt{k+2}]
$$

and

$$
m\left(J^{k}\left(x_{1}, \ldots, x_{m+1}\right), d\right)=\frac{(k+1) \sqrt{ } k+(m-k) \sqrt{ }+2}{(m+1) \sqrt{2 k+2}}
$$

Proof. This is a trivial consequence of Lemma 5 using congruence and scaling.

THEOREM 3. If $X$ is a bounded closed convex subset of $\mathbb{R}^{n}$ and $D$ is the diameter of $X$, then

$$
a((X, d)) \leq D \sqrt{n /(2 n+2)},
$$

and the bound is optimat.

Proof. The bound is established in Lemma 2. To see it is optimal, note that $J\left(x_{1}, \ldots, x_{n+1}\right)$ is a bounded closed convex subset of $\mathbb{R}^{n}$ and $J\left(x_{1}, \ldots, x_{n+1}\right)=J^{n}\left(x_{1}, \ldots, x_{n+1}\right)$. Thus 


$$
a\left(\left\langle J\left(x_{1}, \ldots, x_{n+1}\right), d\right\rangle\right)=D \sqrt{n /(2 n+2)} .
$$

\section{Some related results}

Since this paper was written, Szekeres and Szekeres [6] have independently shown that Stadje's bound is incorrect, and have found Stadje numbers for all compact convex subsets of a normed vector space with real or complex scalars. Their paper "The average distance theorem for compact convex regions" will also appear in the Bulletin of the Australian Mathematical Society, as will a related paper "Average distances in compact connected spaces" by Yost [8].

\section{References}

[1] L.M. Blumenthal and G.E. Wahlin, "On the spherical surface of smallest radius enclosing a bounded subset of $n$-dimensional Euclidean space", Bull. Amer. Math. Soc. 47 (1941), 771-777.

[2] 0. Gross, "The rendezvous value of a metric space", Advances in game theory, 49-53 (Annals of Mathematics Studies, 52. Princeton University Press, Princeton, New Jersey, 1964).

[3] Heinrich Jung, "Ueber die kleinste Kugel, die eine raumliche Figur einschliesst", J. reine Angew. Math. 123 (1901), 241-257.

[4] Sidney A. Morris and Peter Nickolas, "On the average distance property of compact connected metric spaces", Arch. Math. (to appear).

[5] Wolfgang Stadje, "A property of compact connected spaces", Arch. Math. (Basel) 36 (1981), 275-280.

[6] E. Szekeres and G. Szekeres, "The average distance theorem for compact convex regions", Bull. Austral. Math. Soc. (to appear).

[7] D.J. Wilson, "A game with squared distance as payoff", Bull. Austral. Math. Soc. (to appear).

[8] David Yost, "Average distances in compact connected spaces", BuIZ. Austral. Math. Soc. 26 (1982), 331-342.

Department of Pure Mathematics, La Trobe University, Bundoora,

Victoria 3083, Australia. 